\title{
Arguments for tightness testing of nuclear system pipeline parts, attached to pressureless tanks
}

\author{
A I Ivanov ${ }^{1}, A S$ Kritchenkov $^{2,3,4}$ and $M Y u$ Egorov $^{1, *}$ \\ ${ }^{1}$ Peter the Great St.Petersburg Polytechnic University, Polytechnicheskaya 29, St. Petersburg, 195251, Russian Federation \\ ${ }^{2}$ Peoples' Friendship University of Russia (RUDN University), 6 Miklukho-Maklaya Street, Moscow, 117198, Russian Federation \\ ${ }^{3}$ Institute of Macromolecular Compounds of the Russian Academy of Sciences, Bolshoi pr. VO 31, St. Petersburg, 199004, Russian \\ Federation \\ ${ }^{4}$ Saint-Petersburg National Research University of Information Technologies, Mechanics, and Optics, Kronverkskii pr. 49, 197101 St. \\ Petersburg, Russian Federation
}

\begin{abstract}
Actual standard, namely Rules for design and safe operation of equipment and pipelines of nuclear power installations (2015) does not regulate strength and tightness testing of pipeline parts, located between valves and pressureless tanks of nuclear systems at nuclear power plants. This paper introduces arguments for strength and tightness test conduction of such areas of nuclear power plant pipelines, designed in accordance with Russian standards. It was also proposed some amendments for the norm aimed to improve safety and reliability of nuclear power plant systems with radioactive contamination. It was suggested to apply the result of current study to Leningradskaya- 2 in order to improve safety and decrease the number of failures of the liquid radioactive waste storage system.
\end{abstract}

\section{Introduction}

Nuclear power plant (NPP) is a potential source of danger for its operational stuff and people living in the neighborhood. On the other hand, term safety in application to nuclear power plants, means ability to ensure protection for operational stuff and people from harmful factors such as radiation, and maintain the factors below its maximum allowed level within the facility lifecycle and in accordance with federal regulations in the area of atomic energy use [1]. Safety has become the major characteristic, determining acceptance of nuclear technology in general in society. After some well-known nuclear disasters occurred, Chernobyl NPP (1986) and Fukushima-1 NPP (2011), several nations have drastically lost trust in possibility of safe usage of civil nuclear industry [2,3]. Furthermore, Germany has changed national energy policy which resulted in complete termination of new construction of nuclear power plants and switched to energy generation from renewable sources [4].

As of 2019, there are 35 nuclear power units in operation in Russia, and safety of each unit is relied on systems, consisting in general of pipeline and equipment. According to clause 1.2.23 [1], operating organization of nuclear power plant “shall assure NPP's safety including any measures for prevention of accidents and mitigation of their consequences". In order to fulfil the clause, a number of safety tests is carried on within the nuclear power plant lifecycle stages. For instance:
- during the design stage, it is conducted a probabilistic safety analysis (PSA) at several levels such as element-based and functional-based $[5,6]$.

- within the stages, namely construction, commissioning and operation, individual (elementbased) and complex (system-based) tests is executed. The tests may also include subtests such as:

- control of the metal condition for equipment, pipes and its connections aimed on leakage detection [7];

- hydraulic and pneumatic tests in order to justify strength of equipment, absence of leakage, and witness its good conditions and possibility of "operation... until the next regular (planned) control" item 28 [7].

\section{Purpose of the research}

The purpose of this paper is to

- decrease the number of failures caused by leakage from weldings and pipes shells of systems, contacting with radioactive contaminated medium

- facilitate design of new projects of nuclear power plants which constructing in accordance with Russian norms and regulation of atomic energy use.

One of assignments of the research is to eliminate an inconsistence between two norms, namely "Rules for design and safe operation of equipment and pipelines of nuclear power installations" (NP-089-15) [8] and "Safety rules during treatment of radioactive wastes for nuclear power plants" (NP-002-15) [9]. The details of the inconsistence are as follows: the code [8] defines requirements for testing NPP systems. The test

* Corresponding author: mikhail.yu.egorov@gmail.com 
procedure scope, however, does not cover the way of testing of some system elements and pipeline types, namely overflow pipes, hydraulic seal (syphons), and hydrostatic pressure vessels clause 3 item " $n$ " [8].

On the other hand, the code [9] contains requirements for the liquid radioactive waste (LRW) treatment at nuclear power plants. These requirements are as follows:

- the possibility of execution of following operations must be assured while designing the LRW treatment system: visual inspection, renovation, controlling of metal surfaces and weldings after assembling and during operation of the unit clause 15 [9];

- operating organization shall efficiently manage all activities related to operation and maintenance of the radioactive waste management systems aimed to prevent accident clause 51 [9].

The LRW treatment and also storage are processed by the LRW storage system which belongs to 3 safety class and, therefore, the requirements, stated in [9], are applicable to the system.

In summary, several contradicting clauses in codes [8] and [9] limits the scope of the strength and tightness tests of complete systems, making influence on safety, within the two periods: after montage and in operation. These codes must be harmonized in order to improve overall safety of NPP. On second, taking into account that both organizations, designing and operating, have some disagreement upon the way of testing the pipe areas especially within the period of operation of the unit due to the contradiction mentioned above, it is expected that harmonization of codes [8] and [9] will also eliminate the disagreement.

\section{Test technology description and proposal of safety improvements}

In order to confirm readiness as well as strength and tightness of the system elements for exploitation, a set of complex technical examinations must be carried out: once after montage and periodically during operation of the unit. According to [7], the after-montage test must include controlling of metal surfaces and weldings. Subsequent operation is hydraulic (pneumatic) test which must be done periodically according to [8]

The technology of periodical strength and tightness test may be illustrated on example of the intermediate LRW storage system of the unit 1 of Leningradskaya-2 NPP. The system purpose is to store sorbents, mixture of radioactive sewage water and pulp, and process it to the cementation system. In order to avoid radioactive contamination of the room air, the radioactive process blow-offs purification system keeps the LRW storage tank 10KPK10BB001 at slightly below atmosphere pressure. Pipelines, which located between the tank and valves (later named as "non-switchable"), also have the same pressure as the tank. Rosatom uses a code "KPK" for identification of power stations elements in accordance with classification system KKS, which is the abbreviation derived from German term Kraftwerk Kennzeichen system [6] and [11]. All elements of the KPK system received following classification: the " 3 " safety class which is complemented by a symbol " $\mathrm{N}$ ", meaning "normal operation component" according to [1] and the group "C" according to [8]. The fragment of the pipe and instrumentation diagram (P\&ID) of the KPK system is shown in Figure 1.

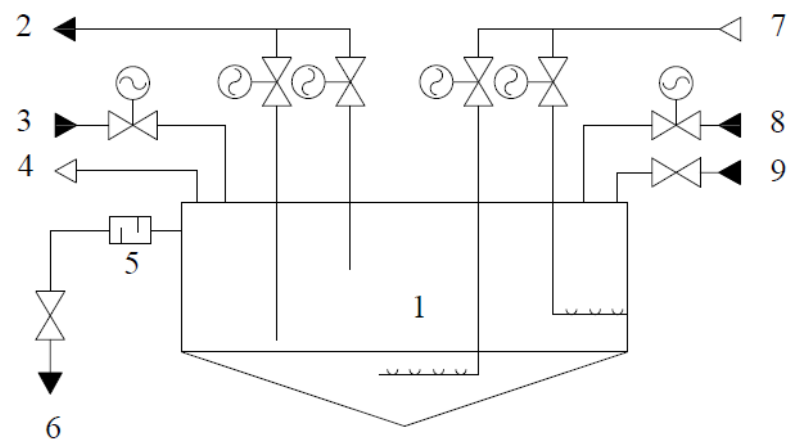

Fig. 1. Fragment of P\&ID diagram of the intermediate liquid radioactive waste storage system of the unit 1 of Leningradskaya-2 NPP: 1 - storage tank 10KPK10BB001, 2 supply pipeline to pumps KPK40; 3 - recirculation pipeline from pipes KPK40; 4 - storage tank vacuuming pipe to the Radioactive process blow-offs purification system (KKS code: KPL-3); 5 - water seal; 6 -drainage; 7 - compressed air supply system (KKS code: QEB); 8 - radioactive pulp supply from concentrated salt producing evaporator KPF30; 9 - wet pulp supply from hydro cyclones KPF12.

Notably, that the number of steps of the test technology significantly alters at different stages of NPP life cycle. Thus, within the period "after montage" test could be logically divided into six steps; that number is bigger by two the number of steps, mentioned in the clause 238 [8]. At the first and the second step, it must be conducted documentation check and visual examination of all system elements respectively. At the third stage, the system must be filled up with the test medium such as air (in pneumatic test) or pure demineralized water (in hydraulic test). This circumstance makes the process:

- fast because water in sufficient amount and quality has been constantly produced at the time for pipeline cleaning, and it can be recycled as well;

- safe for workers since it may harm none in case of leakage.

Then pipelines and tanks, having the same pressure value within the boundaries, must be divided into enclosed contours and then isolated from external pipelines using system valves. At the step four, test pressure must be created in enclosed contour using a compressor or pump in pneumatic or hydraulic test respectively. The pressure value $P_{h}, \mathrm{MPa}$ can be calculated similarly in both tests types according to [8] from following equation:

$$
K_{h} \cdot P \cdot \frac{\sigma^{T_{h}}}{\sigma^{T}}<P_{h}<P_{c r i t},,
$$

where $K_{h}-$ coefficient needed for equalizing the calculation of the tensions $\sigma^{T_{h}}, \sigma^{T}$ in accordance with that of membrane tension. The coefficient value equals 
either 1.25 in case of testing equipment and pipelines, or 1 in case of safety housings and jackets.

$P$ - pressure, MPa which is equal to design pressure in case the test is carried after manufacturing, or operating pressure in case the test is carried after assembling or in the course of operation of NPP;

$\sigma^{T h}, \sigma^{T}$ - rated permissible stresses in metal, MPa, at test temperature $T_{h}$ or at design temperature $T$, respectively;

$P_{\text {crit }}$ - the critical pressure value, at which either total membrane tension equal to $1.35 \cdot \sigma^{T_{h}}$ or sum of total or sum of local membrane and total bending tensions will reach $1.70 \cdot \sigma^{T_{h}}$ in the tested element, $\mathrm{MPa}$.

At the step five, visual inspection of the contour under pressure is carried out. Finally, at the sixth step the system must be returned into its original condition.

All the tanks, working under atmosphere pressure, must be tested separately from system in such way: filled with demineralized water above operational level.

In contrast to test simplicity within the period "after montage", it is much more difficult to conduct hydraulic test during unit operation because of several circumstances. Since some pipelines are filled up with the radioactive technological medium. Thus, it is needed to drain chosen contours prior to test, then to conduct its decontamination. Noticeable, that in some cases is not possible to completely remove contamination from the equipment. Finally, it is needed to fill contours with demineralized water. After completing the test, it is necessary to recycle the test medium from gained radioactivity. These additional operations substantially increase the test difficulty, test duration within the period "during unit operation" and, therefore, exposure doses for the NPP personnel because the system contains many manual valves which could be switched on or off only by operational personnel. Summarizing, hydraulic test within the operational period is much more difficult and labor intensive in comparison with that within the "after montage" period.

On the other hand, it is remarkable that NPP unit operation is determined by many more systems which are in contact with radioactive contaminated medium such as coolant, radioactive floor water, drains, samples; and likewise these systems containing areas with undetermined way of testing. Moreover, a leakage of such contaminated medium from these systems may have similar outcome to that from the LRW treatment system. The outcome, for instance, could be: decontamination of the room surface, localization (i.e. closure of neighboring valves if applicable) of the broken area, drainage of either the system channel or the damaged pipe, repairment of the broken pipe. In the best case, localization can be done easily and refitted due to principles of redundancy and independence of channels; in the worst case, it requires complex changes of operations of the entire system. In total, there are a number of systems which are affected by the contradiction stated above.

Takin into account that metal characteristics, its tendency towards intergranular corrosion, and shell erosion speed are the same for pipelines, equipment, and the pipeline areas with unidentified way of test have the same, it can be suggested to test all system elements, excepting these which is not possible to test, using single method. It can be done, for instance, adding following amendments into code [8]:

- exclude clause 3 item n "hydraulic locks, overflow piping for tanks, hydrostatic pressure vessels";

- paraphrase clause 173: "Within the period "in operation", following items are excluded from the test scope: pipes from sprinklers till the first valve, gravity drains from the last valve till the end of the pipeline having a gravity drain";

- and new clause 173.1: Within the period "in operation", pipeline areas for discharge (supply) of process medium into a tank with atmosphere pressure must be either tested hydraulically or pneumatically or undergo a radiographic control (in case it was made from metal).

On second, it can be suggested to add following amendments into the code [9]:

- paraphrase clause 20 paragraph one: "Design and layout of the radioactive waste treatment equipment and pipelines shall provide the possibility for their inspection, repair, hydraulic (pneumatic) testing, control of metal and weld joints after manufacturing (installation) in accordance with the code "Rules for design and safe operation of equipment and pipelines of nuclear power installations".

\section{Conclusions}

1. This article contains analysis of actual codes, determining hydraulic and pneumatic test technology which affects overall safety of NPP.

2. Some amendments were proposed aiming to eliminate the contradiction between codes: Federal Rules and Regulations in the Field of Nuclear Energy Use. Rules for design and safe operation of equipment and pipelines of nuclear power installations NP-089-15 and Federal Rules and Regulations in the Area of Atomic Energy Use. Safety rules during treatment of radioactive wastes for nuclear power plants NP-002-15.

3. As a result of the norm harmonization, safety of Leningrdskaya-2 NPP has improved and the number of failures of the liquid radioactive waste storage system has decreased because of leakage prevention caused by corrosion.

\section{References}

1. Federal Environmental, Industrial and Nuclear Supervision Service Rostechnadzor 2015 Federal Rules and Regulations in the Area of Atomic Energy Use. General provisions for nuclear power plant safety assurance NP-001-15 (Moscow), 37 (2015)

2. R. Eiser, A. Spears, W. Paul. J. of Applied Social Psychology, 19(8), 689-700 (1989)

3. M. Siegrist, B. Sutterlin, C. Keller, Energy Policy, 69, 356-63 (2014) 
4. A. Energiewende, Understanding the Energiewende, $58(2015)$

5. G. Tokmachev, Izvestiya vuzov. Yadernaya Energetika, 3(I), 44-53 (2007)

6. International Atomic Energy Agency IAEA 2016 Safety of nuclear power plants: design. Specific safety requirements Vienna, 99 (2016)

7. Federal Environmental, Industrial and Nuclear Supervision Service Rostechnadzor 2015 Federal Rules and Regulations in the Area of Atomic Energy Use. Rules for control of base metal, welded joints and deposited surfaces during operation of equipment, pipelines and other components of nuclear power plants NP-084-15 (Moscow), 95 (2015)

8. Federal Environmental, Industrial and Nuclear Supervision Service Rostechnadzor 2016 Federal Rules and Regulations in the Field of Nuclear Energy Use. Rules for design and safe operation of equipment and pipelines of nuclear power installations NP-089-15 (Moscow), 49 (2016)

9. Federal Environmental, Industrial and Nuclear Supervision Service Rostechnadzor 2015 Federal Rules and Regulations in the Area of Atomic Energy Use. Safety rules during treatment of radioactive wastes for nuclear power plants NP002-15 (Moscow), 11 (2015)

10. VGB PowerTech Service GmbH 2011 Letter Code for Power Plant Systems edition 3 (Essen: Publisher technical-schientific documents), 644 (2011)

11. A. Gorbunov, Electricheskiye stantsii, 3, 33-36 (2011) 